\title{
Some Properties of a Kind of Singular Integral Operator with Weierstrass Function Kernel
}

\author{
Lixia Cao \\ Department of Information and Computing Sciences, Mathematics College, Northeast Petroleum University, Daqing, China \\ Email: caolixia98237@163.com
}

Received June 16, 2013; revised July 16, 2013; accepted July 25, 2013

Copyright (C) 2013 Lixia Cao. This is an open access article distributed under the Creative Commons Attribution License, which permits unrestricted use, distribution, and reproduction in any medium, provided the original work is properly cited.

\begin{abstract}
We considered a kind of singular integral operator with Weierstrass function kernel on a simple closed smooth curve in a fundamental period parallelogram. Using the method of complex functions, we established the Bertrand Poincare formula for changing order of the corresponding integration, and some important properties for this kind of singular integral operator.
\end{abstract}

Keywords: Weierstrass Function Kernel; Singular Integral Operator; Bertrand Poincaré Formula; Properties

\section{Introduction}

The properties of singular integral operator with Cauchy or Hilbert kernel on simple closed smooth curve or open arc have been elaborately discussed in [1-3]. Based on these, for the boundary curve is a closed curve or an open arc, the authors discussed the singular integral operators and corresponding equation with Cauchy kernel or Hilbert kernel in [1-3]. In recent years, many authors discussed the numerical solution of a class of systems of Cauchy singular integral equations with constant coefficients, Numerical methods for nonlinear singular Volterra integral equations in [4-6].

In this paper, we consider a kind of singular integral operator with Weierstrass function kernel on a simple closed smooth curve in a fundamental period parallelogram. Our goal is to develop the Bertrand poincare formula for changing order of the corresponding integration, and some important properties of the above singular integral operator.

\section{Preliminaries}

Definition 1 Suppose that $\omega_{1}, \omega_{2}$ are complex constants with $\operatorname{Im}\left(\omega_{1} / \omega_{2}\right) \neq 0$, and $\mathbf{P}$ denotes the fundamental period parallelogram with vertices $\pm \omega_{1} \pm \omega_{2}$. Then the function

$$
\zeta(z)=1 / z+\sum_{m, n}^{\prime}\left[1 /\left(z-\Omega_{m n}\right)+1 / \Omega_{m n}+z / \Omega_{m n}^{2}\right]
$$

is called the Weierstrass $\zeta$-function, where

$$
\Omega_{m n}=2 m \omega_{1}+2 n \omega_{2}
$$

$\sum^{\prime}$ denotes the sum of all $m, n=0, \pm 1, \pm 2, \cdots$, except for ${ }^{m, p} n=n=0$.

Definition 2 Suppose that $L_{0}$ is a smooth closed curve in the counterclockwise direction, lying entirely in the fundamental period parallelogram $\mathbf{P}$, with $z_{0}(\neq 0)$ and the origin lying in the domain $S_{0}^{+}$enclosed by $L_{0}$. The following operator

$$
\begin{aligned}
& K \varphi \equiv a\left(t_{0}\right) \varphi\left(t_{0}\right) \\
& +\frac{1}{\pi i} \int_{L_{0}} \varphi(t) K\left(t_{0}, t\right)\left[\zeta\left(t-t_{0}\right)+\zeta\left(t_{0}-z_{0}\right)\right] \mathrm{d} t, t_{0} \in L_{0}
\end{aligned}
$$

is called the singular integral operator with $\zeta$-function kernel on $L_{0}$, where $\varphi(t) \in H\left(L_{0}\right)$ is the unknown function, and

$$
K\left(t_{0}, t\right) \in H\left(L_{0} \times L_{0}\right), a(t) \in H\left(L_{0}\right)
$$

are the given functions.

Letting $b(t)=K(t, t)$, then (1) becomes

$$
\begin{aligned}
& K \varphi \equiv a\left(t_{0}\right) \varphi\left(t_{0}\right)+\frac{b\left(t_{0}\right)}{\pi i} \int_{L_{0}} \varphi(t)\left[\zeta\left(t-t_{0}\right)+\zeta\left(t_{0}-z_{0}\right)\right] \mathrm{d} t \\
& +\frac{1}{\pi i} \int_{L_{0}}\left[K\left(t_{0}, t\right)-K\left(t_{0}, t_{0}\right)\right]\left[\zeta\left(t-t_{0}\right)+\zeta\left(t_{0}-z_{0}\right)\right] \varphi(t) \mathrm{d} t
\end{aligned}
$$


Since $\zeta(t)$ is uniformly convergent in any closed bounded region lying entirely in $\mathbf{P}$,

$$
\left|\zeta\left(t-t_{0}\right)+\zeta\left(t_{0}-z_{0}\right)\right| \leq 1 /\left|t-t_{0}\right|+M
$$

for any $t_{0}, t \in L_{0}$, where $M$ is some positive finite constant. By noting that $K\left(t_{0}, t\right) \in H^{\alpha}(0<\alpha \leq 1)$, we obtain

$$
\left|\left[K\left(t_{0}, t\right)-K\left(t_{0}, t_{0}\right)\right]\left[\zeta\left(t-t_{0}\right)+\zeta\left(t_{0}-z_{0}\right)\right]\right| \leq N /\left|t-t_{0}\right|^{\lambda}
$$

$(0<\lambda \leq 1)$, where $N$ is some positive finite constant. Write

$$
\begin{gathered}
K^{0} \varphi \equiv a\left(t_{0}\right) \varphi\left(t_{0}\right)+\frac{b\left(t_{0}\right)}{\pi i} \int_{L_{0}} \varphi(t)\left[\zeta\left(t-t_{0}\right)+\zeta\left(t_{0}-z_{0}\right)\right] \mathrm{d} t \\
k\left(t_{0}, t\right)=\frac{1}{\pi i}\left[K\left(t_{0}, t\right)-K\left(t_{0}, t_{0}\right)\right]\left[\zeta\left(t-t_{0}\right)+\zeta\left(t_{0}-z_{0}\right)\right] \quad \text { defined as } \quad \kappa=\frac{1}{2 \pi}\left[\arg \frac{D(t)}{S(t)}\right]_{L_{0}}, \text { where } \\
k \varphi \equiv \int_{L_{0}} k\left(t_{0}, t\right) \varphi(t) \mathrm{d} t \quad S(t)=a(t)+b(t), D(t)=a(t)-b(t)
\end{gathered}
$$

then (1) can be rewritten in the form

$$
\left(K^{0}+k\right) \varphi,
$$

where $k$ is a Fredholm operator and $K^{0}$ is called the characteristic operator of $K$. Now the index of $K$ is

and for definiteness we assume that $a^{2}(t)-b^{2}(t) \neq 0$, namely we assume that $K$ is an operator of normal type.

Now the associated operator of (1) takes the form

$$
K^{\prime} \psi \equiv a\left(t_{0}\right) \psi\left(t_{0}\right)-\frac{1}{\pi i} \int_{L_{0}} K\left(t, t_{0}\right) \psi t\left[\zeta\left(t-t_{0}\right)+\zeta\left(t_{0}-z_{0}\right)\right] \mathrm{d} t, t_{0} \in L,
$$

or

$$
K^{\prime} \psi \equiv a\left(t_{0}\right) \psi\left(t_{0}\right)-\frac{1}{\pi i} \int_{L_{0}} b(t) \psi(t)\left[\zeta\left(t-t_{0}\right)+\zeta\left(t_{0}-z_{0}\right)\right] \mathrm{d} t+\int_{L_{0}} k\left(t, t_{0}\right) \psi(t) \mathrm{d} t, \quad t_{0} \in L,
$$

and so that the associated operator of $K^{0}$ becomes

$$
K^{0 \prime} \psi \equiv a\left(t_{0}\right) \psi\left(t_{0}\right)-\frac{1}{\pi i} \int_{L_{0}} b(t) \psi(t)\left[\zeta\left(t-t_{0}\right)+\zeta\left(t_{0}-z_{0}\right)\right] \mathrm{d} t, t_{0} \in L .
$$

In addition, if we write

$$
k_{1} \psi\left(t_{0}\right)=\int_{L_{0}}\left\{k\left(t, t_{0}\right)-\frac{1}{\pi i}\left[b(t)-b\left(t_{0}\right)\right]\left[\zeta\left(t-t_{0}\right)+\zeta\left(t_{0}-z_{0}\right)\right]\right\} \psi(t) \mathrm{d} t, t_{0} \in L,
$$

then (4) can be rewritten as

$$
K^{\prime} \psi \equiv a\left(t_{0}\right) \psi\left(t_{0}\right)-\frac{b\left(t_{0}\right)}{\pi i} \int_{L_{0}} \psi(t)\left[\zeta\left(t-t_{0}\right)+\zeta\left(t_{0}-z_{0}\right)\right] \mathrm{d} t+k_{1} \psi(t), t_{0} \in L,
$$

where

$$
\left|\left[b(t)-b\left(t_{0}\right)\right]\left[\zeta\left(t-t_{0}\right)+\zeta\left(t_{0}-z_{0}\right)\right]\right| \leq D /\left|t-t_{0}\right|^{\lambda} \quad(0 \leq \lambda<1, \quad D \text { is some finite constant })
$$

So $k_{1} \psi$ is a Fredholm operator, and then the charac- teristic operator of $K^{\prime}$ operator becomes

$$
K^{\prime 0} \psi \equiv a\left(t_{0}\right) \psi\left(t_{0}\right)-\frac{b\left(t_{0}\right)}{\pi i} \int_{L_{0}} \psi(t)\left[\zeta\left(t-t_{0}\right)+\zeta\left(t_{0}-z_{0}\right)\right] \mathrm{d} t
$$

Therefore, we concluded that $K^{\prime 0} \psi=K^{0 \prime} \psi$ usually can not be established, that is $K^{\prime 0} \psi \neq K^{0 \prime} \psi$.

For convenience, we write

$$
\mathrm{X}(z, \sigma)=\zeta(z-\sigma)+\zeta\left(\sigma-z_{0}\right)
$$

where the fixed nonzero point $z_{0}$ and the origin lie in $S_{0}^{+}$. It is not difficult to get the following results.
Lemma 1 Suppose that $f(t, \tau) \in H\left(L_{0} \times L_{0}\right)$, and with the same $L_{0}$ as mentioned before, then

a) $\int_{L_{0}} \mathrm{~d} t \int_{L_{0}} f(t, \tau) \mathrm{X}(\tau, t) \mathrm{d} \tau=\int_{L_{0}} \mathrm{~d} \tau \int_{L_{0}} f(t, \tau) \mathrm{X}(\tau, t) \mathrm{d} t$,

$$
\int_{L_{0}} \mathrm{X}(\tau, t) \mathrm{d} t \int_{L_{0}} f(t, \tau) \mathrm{d} \tau=\int_{L_{0}} \mathrm{~d} \tau \int_{L_{0}} f(t, \tau) \mathrm{X}(\tau, t) \mathrm{d} t
$$


b) (Poincare-Bertrand formula)

$$
\int_{L_{0}} \mathrm{X}\left(t, t_{0}\right) \mathrm{d} t \int_{L_{0}} f(t, \tau) \mathrm{X}(\tau, t) \mathrm{d} \tau=-\pi^{2} f\left(t_{0}, t_{0}\right)
$$

\section{Some Properties of Operator $K$}

1) If $\varphi \in H$, then $K \varphi \in H$.

Proof Through calculation and estimation, we have

$$
\left|\int_{L_{0}} K\left(t_{1}, t\right) \varphi(t) \zeta\left(t_{1}\right) \mathrm{d} t-\int_{L_{0}} K\left(t_{2}, t\right) \varphi(t) \zeta\left(t_{2}\right) \mathrm{d} t\right| \leq M\left|t_{1}-t_{2}\right|^{\alpha}+N\left|\zeta\left(t_{1}\right)-\zeta\left(t_{2}\right)\right|
$$

for any $t_{1}, t_{2} \in L_{0}$, where $M$ and $N$ are all finite constant. While for any $t_{1}, t_{2} \in L_{0}$, we have

$$
\left|\zeta\left(t_{1}\right)-\zeta\left(t_{2}\right)\right| \leq\left|\frac{t_{1}-t_{2}}{t_{1} t_{2}}\right|+\sum_{m, n}^{\prime}\left|\frac{t_{1}-t_{2}}{\left(t_{1}-\Omega_{m n}\right)\left(t_{2}-\Omega_{m n}\right)}\right|+\sum_{m, n}^{\prime}\left|\frac{t_{1}-t_{2}}{\Omega_{m n}^{2}}\right| \leq Q\left|t_{1}-t_{2}\right|
$$

where $Q$ is some finite constant. Substituting (8) into (7), we obtain

$$
\int_{L_{0}} K\left(t_{0}, t\right) \varphi(t) \zeta\left(t_{0}\right) \mathrm{d} t \in H
$$

Similarly we know that

$$
\int_{L_{0}} K\left(t_{0}, t\right) \varphi(t) \zeta\left(t-t_{0}\right) \mathrm{d} t, \int_{L_{0}} K\left(t_{0}, t\right) \varphi(t) \zeta\left(t_{0}-z_{0}\right) \mathrm{d} t, a\left(t_{0}\right) \varphi\left(t_{0}\right) \in H
$$

Consequently, we have $K \varphi \in H$.

2) If $K_{1}, K_{2}$ are singular integral operator, then

$$
K_{j} \varphi \equiv a_{j}\left(t_{0}\right) \varphi\left(t_{0}\right)+\frac{1}{\pi i} \int_{L_{0}} \varphi(t) K_{j}\left(t_{0}, t\right)\left[\zeta\left(t-t_{0}\right)+\zeta\left(t_{0}-z_{0}\right)\right] \mathrm{d} t, j=1,2
$$

then

$$
\begin{aligned}
& K_{1} K_{2} \varphi=\left[a_{1}\left(t_{0}\right) a_{2}\left(t_{0}\right)+b_{1}\left(t_{0}\right) b_{2}\left(t_{0}\right)\right] \varphi\left(t_{0}\right) \\
& +\int_{L_{0}} \frac{\left[a_{1}\left(t_{0}\right) K_{2}\left(t_{0}, t\right)+a_{2}(t) K_{1}\left(t_{0}, t\right)\right]}{\pi i} \varphi(t)\left[\zeta\left(t-t_{0}\right)+\zeta\left(t_{0}-z_{0}\right)\right] \mathrm{d} t \\
& +\frac{1}{(\pi i)^{2}} \int_{L_{0}}\left\{\int_{L_{0}} K_{1}\left(t_{0}, t_{1}\right) K_{2}\left(t_{1}, t\right)\left[\zeta\left(t_{1}-t_{0}\right)+\zeta\left(t_{0}-z_{0}\right)\right]\left[\zeta\left(t-t_{1}\right)+\zeta\left(t_{1}-z_{0}\right)\right] \mathrm{d} t_{1}\right\} \varphi(t) \mathrm{d} t
\end{aligned}
$$

where the sum of the former two terms in the right hand of Equation (10) are the characteristic operator, and the remainder in that is a Fredholm operator.

Proof By definition, we deduce that

$$
\begin{aligned}
& K_{1} K_{2} \varphi=a_{1}\left(t_{0}\right) a_{2}\left(t_{0}\right) \varphi\left(t_{0}\right)+\frac{1}{\pi i} \int_{L_{0}} a_{1}\left(t_{0}\right) K_{2}\left(t_{0}, t\right) \varphi(t)\left[\zeta\left(t-t_{0}\right)+\zeta\left(t_{0}-z_{0}\right)\right] \mathrm{d} t \\
& +\frac{1}{\pi i} \int_{L_{0}} a_{2}(t) K_{1}\left(t_{0}, t\right) \varphi(t)\left[\zeta\left(t-t_{0}\right)+\zeta\left(t_{0}-z_{0}\right)\right] \mathrm{d} t+C\left(t_{0}\right)
\end{aligned}
$$

where

$$
C\left(t_{0}\right)=\frac{1}{(\pi i)^{2}} \int_{L_{0}} K_{1}\left(t_{0}, t\right)\left[\zeta\left(t-t_{0}\right)+\zeta\left(t_{0}-z_{0}\right)\right]\left\{\int_{L_{0}} K_{2}\left(t, t_{1}\right)\left[\zeta\left(t_{1}-t\right)+\zeta\left(t-z_{0}\right)\right] \varphi\left(t_{1}\right) \mathrm{d} t_{1}\right\} \mathrm{d} t
$$

By virtue of Lemma 1 (b), $C\left(t_{0}\right)$ can be rewritten in the form

$$
C\left(t_{0}\right)=b_{1}\left(t_{0}\right) b_{2}\left(t_{0}\right) \varphi\left(t_{0}\right)+\frac{1}{(\pi i)^{2}} \int_{L_{0}}\left\{\int_{L_{0}} K_{1}\left(t_{0}, t_{1}\right) K_{2}\left(t_{1}, t\right)\left[\zeta\left(t_{1}-t_{0}\right)+\zeta\left(t_{0}-z_{0}\right)\right]\left[\zeta\left(t-t_{1}\right)+\zeta\left(t_{1}-z_{0}\right)\right] \mathrm{d} t_{1}\right\} \varphi(t) \mathrm{d} t
$$




$$
\int_{L_{0}} K_{1}\left(t_{0}, t_{1}\right) K_{2}\left(t_{1}, t\right) \zeta\left(t_{1}-t_{0}\right) \zeta\left(t-t_{1}\right) \mathrm{d} t_{1}=\langle 1\rangle+\langle 2\rangle+\langle 3\rangle+\langle 4\rangle
$$

where

$$
\begin{gathered}
\langle 1\rangle=\int_{L_{0}} \frac{K_{1}\left(t_{0}, t_{1}\right) K_{2}\left(t_{1}, t\right)}{\left(t_{1}-t_{0}\right)\left(t-t_{1}\right)} \mathrm{d} t_{1},\langle 2\rangle=\int_{L_{0}} \frac{K_{1}\left(t_{0}, t_{1}\right) K_{2}\left(t_{1}, t\right)}{t_{1}-t_{0}} \sum_{m, n}^{\prime}\left[\frac{1}{t-t_{1}-\Omega_{m n}}+\frac{1}{\Omega_{m n}}+\frac{t-t_{1}}{\Omega_{m n}^{2}}\right] \mathrm{d} t_{1}, \\
\langle 3\rangle=\int_{L_{0}} \frac{K_{1}\left(t_{0}, t_{1}\right) K_{2}\left(t_{1}, t\right)}{t-t_{1}} \sum_{m, n}^{\prime}\left[\frac{1}{t_{1}-t_{0}-\Omega_{m n}}+\frac{1}{\Omega_{m n}}+\frac{t_{1}-z_{0}}{\Omega_{m n}^{2}}\right] \mathrm{d} t_{1}, \\
\langle 4\rangle=\int_{L_{0}} K_{1}\left(t_{0}, t_{1}\right) K_{2}\left(t_{1}, t\right) \sum_{m, n}^{\prime}\left[1 /\left(t-t_{1}-\Omega_{m n}\right)+1 / \Omega_{m n}+\left(t-t_{1}\right) / \Omega_{m n}^{2}\right] \cdot \sum_{m, n}^{\prime}\left[1 /\left(t_{1}-t_{0}-\Omega_{m n}\right)+1 / \Omega_{m n}+\left(t_{1}-t_{0}\right) / \Omega_{m n}^{2}\right] \mathrm{d} t_{1} .
\end{gathered}
$$

By [1], we know that $\langle 1\rangle$ is a Fredholm integral. For $\langle 4\rangle$, we know from

$$
\left.K_{1}\left(t_{0}, t_{1}\right), K_{2}\left(t_{1}, t\right)\right) \in H\left(L_{0} \times L_{0}\right)
$$

that $\langle 4\rangle$ is continuous about the variable $t \in L_{0}$, and so that $\int_{L_{0}}\langle 4\rangle \varphi(t) \mathrm{d} t$ is also a Fredholm integral. By nothing that $\langle 2\rangle$ and $\langle 3\rangle$ have the same form, we only need to discuss either one of them. Here we consider the integral $\langle 2\rangle$. Write

$$
h(z)=\sum_{m, n} \cdot\left[1 /\left(t-z-\Omega_{m n}\right)+1 / \Omega_{m n}+(t-z) / \Omega_{m n}^{2}\right]
$$

then $h\left(t_{1}\right) \in H\left(L_{0}\right)$ is analytic in $\mathbf{P}$ and so that $h\left(t_{1}\right) \in H\left(L_{0}\right)$. Consequently, we read from

$$
K_{1}\left(t_{0}, t_{1}\right), K_{2}\left(t_{1}, t\right) \in H\left(L_{0} \times L_{0}\right)
$$

that $\langle 2\rangle \in H\left(L_{0}\right)$ and so that $\langle 2\rangle$ is continuous on $L_{0}$, therefore $\int_{L_{0}}\langle 2\rangle \varphi(t) \mathrm{d} t$ is also a Fredholm integral.

So far, we conclude that $K_{1} K_{2}$ is a singular integral operator.

3) Let $K_{3}=K_{1} K_{2}$, where $\kappa_{j}$ denotes the indices of $K_{j}(j=1,2,3)$, then $\kappa_{3}=\kappa_{1}+\kappa_{2}$.

Proof From 2), we know

$$
a_{3}=a_{1} a_{2}+b_{1} b_{2}, b_{3}=a_{1} b_{2}+a_{2} b_{1}
$$

and

$$
S_{3}=S_{1} S_{2}, D_{3}=D_{1} D_{2}
$$

so $\kappa_{3}=\kappa_{1}+\kappa_{2}$.

In addition, we can see from

$$
a_{3}=a_{1} a_{2}+b_{1} b_{2} \text { and } b_{3}=a_{1} b_{2}+a_{2} b_{1},
$$

that when $K_{1}, K_{2}$ are normal, $K_{3}$ is also normal.

4) $\left(K_{1} K_{2}\right) K_{2}=K_{1}\left(K_{2} K_{3}\right)$.

5) If $K$ is a singular integral operator, and $k$ is a Fredholm integral operator of the first kind, then $k K$ and $K k$ are also Fredholm integral operators of the first kind.

6) If the indies of $K$ and $K^{\prime}$ are $\kappa$ and $\kappa^{\prime}$ respectively, then $\kappa^{\prime}=-\kappa$.

7) $\left(K_{1} K_{2}\right)^{\prime}=K_{2}^{\prime} K_{1}^{\prime}$.

Through careful calculation, we may obtain 4) - 7).

8) Generally speaking,

$$
\int_{L_{0}} \psi K \varphi \mathrm{d} t=\int_{L_{0}} \varphi K^{\prime} \psi \mathrm{d} t
$$

can not be established for $\varphi, \psi \in H$.

Proof By definition and calculation, we have

$$
\begin{aligned}
\int_{L_{0}} \psi K \varphi \mathrm{d} t & =\int_{L_{0}} \psi t\left\{a(t) \varphi(t)+\frac{1}{\pi i} \int_{L_{0}} K\left(t, t_{1}\right) \varphi\left(t_{1}\right)\left[\zeta\left(t_{1}-t\right)+\left(t-z_{0}\right)\right] \mathrm{d} t_{1}\right\} \mathrm{d} t \\
& =\int_{L_{0}} a(t) \varphi(t) \psi(t) \mathrm{d} t+\frac{1}{\pi i} \int_{L_{0}} \psi(t) \int_{L_{0}} K\left(t, t_{1}\right) \varphi\left(t_{1}\right)\left[\zeta\left(t_{1}-t\right)+\zeta\left(t-z_{0}\right)\right] \mathrm{d} t_{1} \mathrm{~d} t
\end{aligned}
$$

Whereas

$$
\int_{L_{0}} \varphi K^{\prime} \psi \mathrm{d} t=\int_{L_{0}} a(t) \varphi(t) \psi(t) \mathrm{d} t-\frac{1}{\pi i} \int_{L_{0}} \varphi(t) \int_{L_{0}} K\left(t_{1}, t\right) \psi\left(t_{1}\right)\left[\zeta\left(t_{1}-t\right)+\zeta\left(t-z_{0}\right)\right] \mathrm{d} t_{1} \mathrm{~d} t
$$

Let

$$
W=\int_{L_{0}} \varphi(t) \int_{L_{0}} K\left(t_{1}, t\right) \psi\left(t_{1}\right)\left[\zeta\left(t_{1}-t\right)+\zeta\left(t-z_{0}\right)\right] \mathrm{d} t_{1} \mathrm{~d} t
$$

then by Lemma 1(a), we have

$$
\begin{aligned}
W & =\int_{L_{0}}\left\{\int_{L_{0}} K\left(t_{1}, t\right) \varphi(t) \psi\left(t_{1}\right)\left[\zeta\left(t_{1}-t\right)+\zeta\left(t-z_{0}\right)\right] \mathrm{d} t\right\} \mathrm{d} t_{1} \\
& =-\int_{L_{0}} \psi(t)\left\{\int_{L_{0}} K\left(t, t_{1}\right) \varphi\left(t_{1}\right)\left[\zeta\left(t_{1}-t\right)-\zeta\left(t_{1}-z_{0}\right)\right] \mathrm{d} t_{1}\right\} \mathrm{d} t
\end{aligned}
$$


Substituting (13) into (12), we see that

$$
\left.\int_{L_{0}} \varphi K^{\prime} \psi \mathrm{d} t=\int_{L_{0}} a(t) \varphi(t) \psi t \mathrm{~d} t+\frac{1}{\pi i} \int_{L_{0}} \psi(t)\left\{\int_{L_{0}} K\left(t, t_{1}\right)\right) \varphi\left(t_{1}\right)\left[\zeta\left(t_{1}-t\right)-\zeta\left(t_{1}-z_{0}\right)\right] \mathrm{d} t_{1}\right\} \mathrm{d} t .
$$

Therefore, $\int_{L_{0}} \psi K \varphi \mathrm{d} t=\int_{L_{0}} \varphi K^{\prime} \psi \mathrm{d} t$ cannot be established.

\section{REFERENCES}

[1] N. I. Muskhelishvili, "Singular Integral Equations," World Scientific, Singapore City, 1993.

[2] J. K. Lu, "Boundary Value Problems for Analytic Functions," World Scientific, Singapore City, 1993.

[3] F. D. Gakhov, "Boundary Value Problems," Fizmatgiz, Moscow, 1977.

[4] M. C. De Bonis and C. Laurita, "Numerical Solution of Systems of Cauchy Singular Integral Equations with Constant Coefficients," Applied Mathematics and Computa- tion, Vol. 219, No. 4, 2012, pp. 1391-1410. doi:10.1016/j.amc.2012.08.022

[5] T. Diogo and M. Rebelo, "Numerical Methods for Nonlinear Singular Volterra Integral Equations," AIP Conference Proceedings, Kos, 19-25 September 2012, pp. 226-229.

[6] C. Laurita, "A Quadrature Method for Cauchy Singular Integral Equations with Index -1," IMA Journal of Numerical Analysis, Vol. 32, No. 3, 2012, pp. 1071-1095. doi:10.1093/imanum/drr032 